\title{
Real-time communication in IEEE 802.11s mesh networks: simulation assessment considering the interference of non-real-time traffic sources
}

\author{
Carlos M D Viegas $^{1 *}$, Francisco Vasques ${ }^{1}$, Paulo Portugal ${ }^{1}$ and Ricardo Moraes ${ }^{2}$
}

\begin{abstract}
With the widespread deployment of wireless mesh networks (WMNs) in industrial environments, real-time (RT) communication may benefit from the multi-hop relaying infrastructure provided by WMNs. However, RT communication must be able to coexist with non-RT traffic sources that will interfere with RT communication. Within this context, this paper assesses the impact of interferences caused by non-RT traffic sources upon RT traffic in IEEE $802.11 \mathrm{~s}$ mesh networks. Through an extensive set of simulations, we assess the impact of external traffic sources upon a set of RT message streams in different communication scenarios. According to the simulation results, we infer that RT traffic in $802.11 \mathrm{~s}$ networks may be highly affected by external interferences, and therefore, such interferences must be taken into account when setting-up $802.11 \mathrm{~s}$ networks. By varying the network load imposed by external interferences, we provide some useful hints about utilization thresholds above which the network can no longer reliably support RT traffic. We also present insights about the setting-up of some network parameters in order to optimize the RT communication performance.
\end{abstract}

Keywords: Real-time communication; Quality of service; Wireless mesh networks; IEEE 802.11s; Simulation assessment

\section{Introduction}

The IEEE 802.11 family of wireless protocols became the dominant solution for wireless local area networks (WLANs) due to its high performance, low cost, and fast deployment characteristics [1]. Along its increasing popularity, there was also a demand for sharing common services among different devices connected to the network. Wireless mesh networks (WMNs) appeared as a promising approach to deal with heterogeneity and diversity of wireless networks, by introducing multi-hop forwarding at medium access control (MAC) level and allowing wireless interconnection of multiple access points [2].

WMNs provide greater flexibility, reliability, and performance when compared to traditional wireless networks, since they are able to extend network coverage without any additional infrastructure by using multi-hop communication, where nodes can relay traffic by traversing multiple hops to reach a destination [2].

*Correspondence: viegas@fe.up.pt

${ }^{1}$ IdMEC/INEGI/INESC-Tec - Faculdade de Engenharia da Universidade do

Porto, Rua Dr. Roberto Frias, s/n, 4200-465 Porto, Portugal

Full list of author information is available at the end of the article
Packet forwarding in WMNs may be implemented at layer 3 or layer 2. In the former, which is the most common WMN implementation, packet forwarding is performed at network layer by means of internet protocol (IP). In the latter, frame forwarding is performed at data link layer, being the MAC addresses used to deliver frames through the WMN backbone [3]. The IEEE 802.11s standard specifies a layer $2 \mathrm{WMN}$ aiming to extend the coverage of traditional 802.11 WLANs and to allow the support of a larger diversity of wireless technologies [4].

There are several application domains where WMNs can be applied, such as home and enterprise networks, transportation and real-time (RT) systems, and building automation and metropolitan area networks [5]. In this paper, we are particularly interested in the support of RT applications using WMNs.

RT applications are usually not resilient to delay and jitter constraints. Therefore, the deployment of RT services over WMNs requires the use of quality of service (QoS) mechanisms. Most of current RT applications require a priori reservation of network resources (e.g., link bandwidth, time slots, and channels) in order to meet QoS 
requirements. Within this context, several recent research efforts targeted RT communication support and resource reservation techniques over multi-hop networks [6-14].

Most part of the proposed resource reservation techniques are focused on the RT traffic itself, regardless of the interference of non-RT traffic sources. Usually, authors focus on the proposed mechanisms and their performance, and, in some cases, relevant simplifications are made (e.g., ideal wireless channel conditions [15]). Consequently, the related assessments may not reflect the real-world behavior, which is usually prone to interference of non-RT traffic sources that may impact in the communication performance, as highlighted in [16-18].

Within this context, the main goal of this work is to provide a useful insight upon the impact of interfering traffic over RT communication in single-channel IEEE 802.11s networks. To do so, a simulation assessment was carried out using network simulator 3 (ns-3) and considering real-world scenarios. Non-RT traffic flows from different sources were injected in the network in order to evaluate their impact over RT communication performance. This evaluation indicates that resource reservation techniques must consider the impact of the external traffic interference in order to maintain response times of RT traffic under acceptable thresholds. Otherwise, the communication services may not be able to fulfill the expected RT requirements of the supported applications.

This work extends a performance assessment previously presented in [16]. Such work evaluated the communication performance under periodic interference in a mesh-based network. This work introduces a new realistic interference model, with aperiodic and bursty traffic, in a 802.11s WMN. Therefore, more realistic communication scenarios have been considered.

The remainder of this paper is organized as follows. Section 2 presents an overview of IEEE 802.11s standard by describing its main functionalities. Section 3 presents some related work on MAC performance analysis and resource reservation techniques. Section 4 formulates the problem to be evaluated in this paper. Section 5 describes the simulation scenarios used for this evaluation. Section 6 presents an analysis of the results. Finally, Section 7 concludes the paper.

\section{IEEE 802.11s overview}

As an amendment to the IEEE 802.11 standard, the IEEE 802.11s WMN standard uses the same physical (PHY) layer specification and MAC sublayer architecture, with additional extensions [4]. It introduces forwarding at MAC level that uses a multi-hop wireless relaying infrastructure, where nodes cooperatively maintain the network connectivity. Every node can work as a relaying node, forwarding frames in behalf of its neighbor nodes.
The mesh connectivity is managed by the mesh peering management (MPM) protocol, which is responsible to establish, manage, and tear down mesh peer links among mesh stations (STAs).

The default path selection protocol is the hybrid wireless mesh protocol (HWMP), which combines reactive (ondemand) path selection with extensions to enable proactive (tree-based) path selection. The reactive mode is based on the ad hoc on-demand distance vector (AODV) routing protocol, which allows mesh STAs to communicate in a peer-to-peer basis [19]. In the proactive mode, additional tree building functionality is added to the ondemand mode, by configuring a mesh STA as root of a path tree (formally root mesh STA). The root is responsible to coordinate the path selection by periodically sending proactive information elements to the mesh STAs.

HWMP uses radio-aware metrics, being the airtime link metric the default one. The airtime link metric is proposed for basic interoperability among 802.11 s devices and reflects the amount of channel resources consumed during a frame transmission over a particular link. The path with smallest airtime link metric is considered to be the best forwarding path [20].

The medium access control is managed by the mesh coordination function (MCF), which schedules the access to the channel by allocating transmission opportunities (TXOPs) to mesh STAs. A TXOP is a time-bounded interval in which a station keeps the medium access control [17]. MCF adopts the enhanced distributed channel access (EDCA) as the mandatory MAC scheme, which is a contention-based channel access mechanism based on carrier sensing multiple access with collision avoidance (CSMA/CA).

EDCA provides service differentiation by classifying frames from upper layers in different access categories (ACs). There are four defined ACs, in which frames of different traffic types are mapped according to the application and its QoS requirements. These ACs are based on eight priority levels of IEEE 802.1D standard, as follows [21]: background (BK), best effort (BE), video (VI), and voice $(\mathrm{VO})$ traffic.

For each $\mathrm{AC}$, an enhanced variant of the distribution coordination function (DCF), called EDCA function (EDCAF), contends for TXOPs using a set of EDCA parameters. These EDCA parameters modify the backoff process with individual interframe spaces and contention windows (CWs) per AC (see Table 1).

The values of $\mathrm{aCW}_{\min }$ and $\mathrm{aCW}$ max, which are the minimum and maximum size of $\mathrm{CW}$, respectively, are defined according to the physical standard in use. For IEEE $802.11 \mathrm{a} / \mathrm{g} / \mathrm{n}$ standards, these values are respectively 15 and 1,023 and for IEEE 802.11b are 31 and 1,023. Whenever in the presence of IEEE $802.11 \mathrm{~b}$ devices, the IEEE $802.11 \mathrm{~g}$ standard defines 31 and 1,023 values for 
Table 1 Default DCF and EDCA parameter set

\begin{tabular}{|c|c|c|c|c|c|}
\hline Parameters & $A C$ & $\mathrm{CW}_{\min }$ & $\mathrm{CW}_{\max }$ & AIFSN & DIFS \\
\hline \multirow[t]{2}{*}{ DCF } & - & $\mathrm{aCW}$ min & $\mathrm{aCW}$ max & - & 2 \\
\hline & AC_BK & $\mathrm{aCW}_{\min }$ & $\mathrm{aCW}$ max & 7 & - \\
\hline \multirow{3}{*}{ EDCA } & AC_BE & $\mathrm{aCW}$ min & $\mathrm{aCW} \max$ & 3 & - \\
\hline & $A C \_V I$ & $(\mathrm{aCW}$ min +1$) / 2-1$ & $\mathrm{aCW}$ min & 2 & - \\
\hline & AC_VO & $\left(\mathrm{aCW} W_{\min }+1\right) / 4-1$ & $\left(\mathrm{aCW} W_{\min }+1\right) / 2-1$ & 2 & - \\
\hline
\end{tabular}

$\mathrm{aCW}_{\min }$ and $\mathrm{aCW} W_{\max }$, respectively, in order to maintain the compatibility between standards [4].

During the contention phase, each station senses the medium in order to start the frame transmission. If the medium is idle for at least one arbitration interframe space (AIFS[AC]), the station transmits its frames. Otherwise, the station initiates a backoff interval in order to avoid collisions. The duration of AIFS[AC] is given by:

$$
\text { AIFS }[\text { AC }]=\text { AIFSN }[\text { AC }] \times \text { aSlotTime }+ \text { aSIFSTime, }
$$

where AIFSN[AC] defines the number of slot times per AC (AIFSN $[A C] \geq 2$ ), aSlotTime is the slot duration and aSIFSTime is the short interframe space (SIFS) duration.

The backoff time is a uniformly distributed value between zero and the size of CW. At each unsuccessful transmission, the size of $\mathrm{CW}$ is exponentially increased until it reaches the maximum $\mathrm{CW}$ size $\left(\mathrm{aCW}_{\max }\right)$. The $\mathrm{CW}$ size is given by:

$\mathrm{CW}[\mathrm{AC}]=\min \left(2 \times \mathrm{aCW}_{\min }[\mathrm{AC}]+1, \mathrm{aCW}_{\max }[\mathrm{AC}]\right)$.

Once a station has started its backoff time, $\mathrm{CW}$ is decremented every slot time. If the medium becomes busy during the backoff, the station pauses the countdown procedure, which will be resumed only when the medium becomes idle again during an AIFS[AC]. The station will only be able to transmit data when its backoff time reaches zero.

If the backoff time of two or more ACs in the same station reaches simultaneously zero, a virtual collision will occur. In this case, the AC with the higher priority will transmit, whereas all other ACs will act as if a collision occurred in the medium.

In addition, there is a request to send/clear to send (RTS/CTS) scheme to solve the hidden terminal problem. This problem occurs when a station is able to receive the signal from two different neighbors, but those neighbors cannot detect the signal from each other. This is an optional mechanism that operates by exchanging RTS and CTS control frames. When a transmitter needs to send its data, it requests the medium usage by sending a RTS frame and waits for a response from the receiver in form of a CTS frame, informing idle medium. If a CTS frame is not received after a RTS, the transmitter starts a backoff time before retransmitting the RTS frame.
The MCF also defines an optional MAC scheme called MCF controlled channel access (MCCA) [4]. It is a collision-free and guaranteed channel access for QoSaware traffic during reserved periods. MCCA allows mesh STAs to access the channel during predefined intervals with lower contention parameters. It operates alongside EDCA, where a mesh STA obtains a MCCA-TXOP instead of a EDCA-TXOP. Nevertheless, the focus of this paper is upon WMNs that use the EDCA scheme, which is the mandatory MAC for IEEE 802.11s WMNs.

\section{Related work}

The EDCA mechanism was originally proposed in the IEEE 802.11e standard to reduce the number of occurring collisions at MAC sublayer. Its underlying idea was previously proposed by Deng and Chang in [22]. A set of priority classes are defined, where the higher priority class uses the window $\left[0,2^{j+1}-1\right]$ and the lower priority class uses the window $\left[2^{j+1}, 2^{j+2}-1\right]$, where $j$ is the backoff stage. As the EDCA mechanism provides four access categories for traffic differentiation, it would be expected that the highest access category (voice) would be adequate to transfer RT traffic. However, some research papers, analyzing single-hop networks, show that default parameter values of EDCA mechanism are just able to guarantee RT requirements for a smaller number of stations with large message stream periods [17].

In analyzing research papers, there are several analytical models that evaluate the EDCA mechanism in single-hop networks [23-28]. However, the majority of these models assume simplified approaches. Common examples of these simplifications are related to the modeling of the AIFS procedure, backoff counter, TXOP, virtual collisions, and retransmission limits. Besides, most of the analytical models presented in the literature assume that the network operates in saturated traffic conditions.

There is also a number of proposals aiming to improve the EDCA mechanism. The assessment presented in [17] shows that EDCA contention parameters play an important role in the communication, where by adjusting them, it is possible to improve the communication performance. In [29], the authors propose to not double the contention window size in the case of a virtual collision that is not followed by a real collision, where the backoff time is shortened and the traffic is only penalized if it collides during the medium access (i.e., a real collision). In [30], contention parameters can be adapted based on the transmission success ratio aiming to reduce the backoff time. If the success ratio is higher, shorter values for CW, AIFS, and TXOP are used. On the contrary, if the success ratio worsens, the contention parameter values are reverted to their default values. In [31], it is proposed a new scheme to adjust the contention window size based on the queue occupancy. If the queue occupancy is greater 
than a threshold, the CW size is increased by a determined factor.

Concerning the coexistence of EDCA and DCF mechanisms in single-hop networks, the main results show that EDCA mechanism with AIFSN $=2$ (default value defined to the voice access category) presents better performance over DCF stations specially for high priority traffic [32,33]. This specific behavior is a consequence of the different slot decrementing mechanism when compared to the DCF access method.

There is also a set of papers assessing the EDCA scheme in multi-hop networks, where it presents a poor performance due to throughput degradation as the number of hops increases and also due to the hidden terminal problem, which increases the collision probability $[34,35]$. In addition, as presented in [36,37], RTS/CTS mechanism does not improve the network communication performance when considering an ad hoc/mesh network. Likewise, in [38], it is shown that RTS/CTS mechanism only increases the network overhead.

Despite the MCF scheme defined in the IEEE 802.11s, several resource reservation techniques have been also proposed for IEEE 802.11/11s networks. Resource reservation consists of ensuring enough bandwidth and/or channel access opportunities for RT traffic, in order to guarantee its QoS requirements. The following paragraphs summarize the most interesting and relevant techniques for this purpose, focusing on how RT traffic is modeled and how network interference is considered.

The EDCA with resource reservation (EDCA/RR) was proposed to improve EDCA by allowing resource reservation [6]. It extends the EDCA mechanism by adding distributed resource reservation, admission control, and scheduling. Whenever a station wants to perform a reservation, it must broadcast a request and its QoS requirements must be known by the routing protocol. Resources may only be reserved by high priority traffic, whereas the low priority traffic is processed according to EDCA's admission control. A disadvantage of EDCA/RR is that QoS requirements must be known in advance by the routing protocol. Despite considering an interfering traffic pattern with specific payloads and periods, the authors did not evaluate its impact over the RT communication itself.

A similar approach to EDCA/RR is proposed in [7]. Stations can reserve resources by sending requests, but their neighbors must be informed about future transmissions in order to avoid collisions. This technique itself does not have any admission control nor any traffic differentiation scheme, which turns it unable to provide QoS guarantees [6]. Thus, it is not able to prevent interfering traffic from colliding with frames for which resources were reserved.
In [8], the authors proposed the active neighbor bandwidth reservation (AC-ANBR) as a bandwidth reservation technique. RT traffic is admitted by guaranteeing QoS for all message streams based on active neighbor bandwidth. The proposed technique estimates the available bandwidth of each node and the required bandwidth of each new message stream in order to avoid RT traffic from overusing the available bandwidth resources. Despite the enhancement of the network bandwidth usage, the authors did not consider the impact of interference traffic over the RT communication.

The distributed end-to-end allocation of time slots for real-time (DARE) protocol is a scheme that performs endto-end reservations for RT traffic [10]. It operates at MAC sublayer by periodically reserving time slots in nodes along a path. It employs a RTS/CTS-based scheme to perform end-to-end time slot reservations. This protocol offers reliable and efficient support for QoS applications, by providing constant throughput and lower and stable end-to-end delay for a reserved RT message stream. The main disadvantage of DARE is the complex and inefficient mechanism for multiple reservations, where a requested reservation may conflict with previously existing ones [6].

Timestamp-ordered MAC (TMAC) is a MAC protocol that aims to improve packet scheduling fairness in WMNs [13]. TMAC measures packet age by means of timestamps and considers it as the metric for prioritization. These timestamps enforce a local ordering among neighboring nodes. TMAC employs a polling scheme by means of modified RTS/CTS control frames. A transmitter polls its neighboring nodes in a parentchild relationship, seeking to confirm if they do not have older packets awaiting for transmission. This polling scheme ensures that a node cannot starve its children at the cost of its own transmission. Despite the improved performance regarding the resource allocation in the network, TMAC only performs the local ordering considering its adjacent neighbors. Consequently, non-adjacent nodes may still interfere in the scheduling scheme and degrade its performance, since RTS/CTS can suffer from unpredictable delays of uncontrolled traffic in the network.

Regarding the optional MCCA scheme, although being able to provide prioritized medium access for RT traffic, Krasilov et al. in [11] have shown that it may suffer from the external interference impact since the non-MCCA mesh STAs are not aware of MCCA reservations. The authors proposed an improved reservation allocation vector (RAV) setup called directional RAV (DRAV) in order to avoid the problem of ACK-induced interference, which consists of a collision between an ACK frame and a reservation. The DRAV scheme forbids a mesh STA to start an EDCA-TXOP if it crosses any MCCA reservation. 
As MCCA only performs single-hop reservations, the reservation-based HWMP (R-HWMP) has been proposed as a bandwidth reservation protocol that performs end-to-end reservations among several mesh STAs [14]. R-HWMP modifies the HWMP control frames by introducing some of the flow specification concepts of resource reservation protocol (RSVP) [39]. In the path discovery procedure, the R-HWMP evaluates the number of required slots for each flow transmitted from a specific source. Then, it uses the slot information to find available paths from the source to the destination. Nevertheless, this technique may also suffer from the impact of uncontrolled interferences, since the required slots may be unavailable at the moment of path discovery or frames transmission.

As it can be drawn from the aforementioned works, most of the authors do not consider the impact of interfering traffic over the RT communication behavior. A relevant exception is the work presented in [11], where a performance assessment similar to the one done in this work is presented. The main difference is that in [11], the authors define the prioritized traffic to be transmitted using the optional MCCA, whereas the interference traffic is transmitted using EDCA background class. The evaluation assessment shows that MCCA scheme is impacted by the coexistence of EDCA traffic, due to the ACK-induced interference.

Contrarily to the work presented in [11], the main motivation for this work is to assess the performance of the standard EDCA scheme by itself when the medium is shared between prioritized traffic and non-RT traffic in a WMN, both supported by stations implementing the EDCA mechanism, as defined in the standard. We aim to identify the most relevant EDCA limitations in what concerns the support of RT message streams in real-world WMNs.

\section{Problem formulation}

We assess the behavior of a mesh network when RT traffic (traffic generated by high priority applications) and HTTP traffic (interference traffic) share the same wireless channel. The goal of this work is to assess how the network can reliably support RT communication under this mixed traffic condition.

Four RT message streams were considered in a mesh communication scenario with small fixed-sized messages of 80 and 300 bytes and constant periodicities of 50 and $200 \mathrm{~ms}$. The deadlines of RT message streams were considered equal to the periods.

The non-RT interference traffic was modeled to mimic a hypertext transfer protocol (HTTP) conversation [40]. It works as a request-response protocol in the client-server computing model. Clients send requests to a server, which returns responses with the requested content. Usually, the requested content contains several objects (e.g., images, text, videos, or audio). Thus, once a server receives a request, it answers with one or multiple objects, which constitute several bursts of data.

HTTP traffic was divided into sessions with active and inactive periods, which represent webpage downloads and intermediate reading times. The reading times were considered as the interval between client requests.

To mimic the HTTP conversation behavior, it was considered a client side and a server side. Table 2 summarizes the parameters used to define the HTTP traffic model. The HTTP traffic uses the transmission control protocol (TCP) as the transport-layer protocol.

The client side was characterized by request size and request interval parameters. According to the literature [41-43], the typical mean size of requests is 300 bytes, varying from 10 to 2,500 bytes. A truncated lognormal distribution was used to represent this interval. The mean request interval depends on the user-client behavior. A user can request a page and spend a considerable amount of time until making a new request or it can request several pages in a short interval. This behavior was modeled according to a Poisson process with a selectable mean value, i.e., during the simulation process, this mean value will be manually selected according to the desired interference load.

Table 2 HTTP traffic parametrization

\begin{tabular}{|c|c|}
\hline Parameter & Statistical characterization \\
\hline Client request size & $\begin{array}{l}\text { Truncated log-normal distribution: } \\
\mu=5.61, \sigma=0.47 \\
\text { mean }=300 \text { bytes } \\
\text { min }=10 \text { bytes, } \max =2,500 \text { bytes }\end{array}$ \\
\hline Client request interval & $\begin{array}{l}\text { Truncated Poisson process: } \\
\text { mean }=\text { variable } \\
\max =30 \mathrm{~s}\end{array}$ \\
\hline Server response object size & $\begin{array}{l}\text { Truncated log-normal distribution: } \\
\mu=6.17, \sigma=2.36 \\
\text { mean }=7,800 \text { bytes } \\
\text { min }=50 \text { bytes, } \max =2 \text { Mbytes }\end{array}$ \\
\hline $\begin{array}{l}\text { Number of objects per } \\
\text { server response }\end{array}$ & $\begin{array}{l}\text { Truncated Pareto distribution: } \\
\text { mean }=5.64 \\
\min =2 \text { objects, } \max =50 \text { objects }\end{array}$ \\
\hline Server response delay & $\begin{array}{l}\text { Truncated Poisson process: } \\
\lambda=7.69 \\
\text { mean }=130 \mathrm{~ms} \\
\max =250 \mathrm{~ms}\end{array}$ \\
\hline
\end{tabular}


The server side was characterized by a number of objects and their size and response delay parameters. According to [43], the number of objects was represented by a truncated Pareto distribution with mean of 5.64, being 2 the minimum number of objects per page and 50 the maximum. The size of each object has a mean of 7,800 bytes and varies from 50 bytes to 2 Mbytes. A lognormal distribution was used to represent this interval. The server response delay (i.e., parsing time) was modeled by a Poisson process with a mean of $130 \mathrm{~ms}$.

To summarize the HTTP traffic model, a client sends requests varying from 10 to 2,500 bytes according to a specified periodicity (modeled by a Poisson process) and once the server receives a request, it responds after approximately $130 \mathrm{~ms}$ with bursts of multiple objects varying from 50 bytes to 2 Mbytes.

To assess if the WMN can reliably support RT traffic in the presence of HTTP interference traffic, we consider that at least $85 \%$ of deadlines must be met. If the ratio of deadline misses is greater than $15 \%$, the WMN is considered as not being able to support RT communication. This threshold is called deadline miss threshold (DMT).

For such purpose, two different simulation assessments were considered: 1 ) with maximum priority traffic separation - where real-time traffic is defined to be transmitted at the highest EDCA priority class voice, while the non$\mathrm{RT}$ interference traffic is transmitted at the lowest EDCA priority class background; 2) with mixed priority traffic separation - where real-time traffic is kept at the same class voice, but the non-RT interference traffic is transmitted using multiple priority classes: $30 \%$ is transmitted using voice, $30 \%$ video and $40 \%$ background classes.

Finally, a sensitivity analysis of the WMN behavior has been made in order to analyze the effects of varying EDCA contention and HWMP routing protocol parameters. The target of this third simulation assessment is to provide some useful insights on the setting-up of specific protocol parameters and to assess the impact of such parametrization upon the real-time behavior of the WMN.

This set of simulations is a step forward to the assessment of the capability of IEEE 802.11s WMNs to support real-time communication when the wireless channel is shared with non-RT traffic sources.

\section{Simulation scenarios}

A square grid topology with 25 stationary stations disposed in an area of $128 \times 128 \mathrm{~m}$ was considered to create a mesh network scenario (Figure 1). A stationary grid was selected in order to guarantee that the network is well-balanced.

Considering a grid topology, it is important to determine the combination of distance among nodes, which

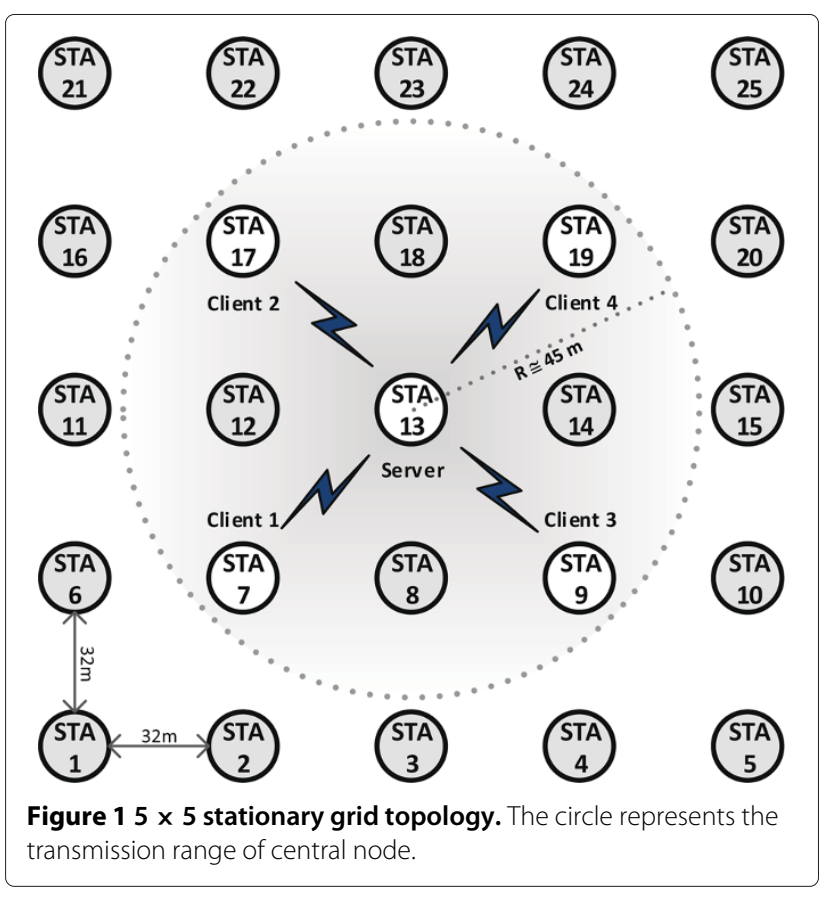

defines the grid density, and the PHY parameters, as antenna gain, data rate, and transmission power. If these parameters are not correctly specified, the mesh network will not be able to be established or it will suffer from instability issues.

If the mesh network is too dense, i.e., if mesh STAs are placed too close from each other, the packet collision rate may become so high that mesh traffic will suffer from unpredictable delays and losses. On the other hand, if the mesh network is too sparse, peer links may become unstable or never established. Besides, if these parameters allow a mesh STA to directly communicate with non-adjacent neighbors, peer links that may be established with that neighbors may become unstable and increase the mesh network traffic, once the links will be frequently opened and closed.

Based on this argumentation, the antenna gains of mesh STAs in the grid were defined to reach just their adjacent neighbors in order to avoid mesh peering instability due to a higher network density. For such purpose, all mesh STAs operate in IEEE 802.11g standard, with the PHY/MAC parameters as defined in Table 3.

The evaluated scenarios consider four RT message streams traversing the mesh network. These streams use user datagram protocol (UDP) as the transport-layer protocol. Different message sizes and periodicities, as well as different sources and destinations, were selected in order to evaluate their behavior in different scenarios. Table 4 presents the RT message streams definition.

In what concerns the interference model, five mesh STAs were defined to implement the HTTP traffic model, 
Table 3 IEEE 802.11g PHY/MAC parameters

\begin{tabular}{|c|c|c|}
\hline Parameter & Description & Value \\
\hline Data rate & $\begin{array}{l}\text { Constant data rate } \\
\text { (no rate adaptation) }\end{array}$ & $24 \mathrm{Mbps}$ \\
\hline Basic rate & - & $6 \mathrm{Mbps}$ \\
\hline Channel number & Fixed channel number & $6(2.437 \mathrm{GHz})$ \\
\hline Channel width & - & $20 \mathrm{MHz}$ \\
\hline $\begin{array}{l}\text { Energy detection } \\
\text { threshold }\end{array}$ & - & $-96 \mathrm{dBm}$ \\
\hline Cca mode 1 threshold & $\begin{array}{l}\text { Clear channel assessment } \\
\text { threshold }\end{array}$ & $-99 \mathrm{dBm}$ \\
\hline Antenna type & - & Omnidirectional \\
\hline Tx/Rx gain & $\begin{array}{l}\text { Antenna gain for } \\
\text { transmission/reception }\end{array}$ & $1.0 \mathrm{dBi}$ \\
\hline Tx power & Transmission power level & $\begin{array}{l}16.0206 \mathrm{dBm} \\
(40 \mathrm{~mW})\end{array}$ \\
\hline Rx noise figure & $\begin{array}{l}\text { SNR degradation in the } \\
\text { receiver }\end{array}$ & $7 \mathrm{~dB}$ \\
\hline $\begin{array}{l}\text { Propagation loss } \\
\text { model }\end{array}$ & - & Log-distance \\
\hline Error rate model & - & Nist OFDM $[44,45]$ \\
\hline $\mathrm{aCW}_{\min }$ & $\begin{array}{l}\text { Minimum contention } \\
\text { window size }\end{array}$ & 15 \\
\hline $\mathrm{aCW} \mathrm{max}_{\max }$ & $\begin{array}{l}\text { Maximum contention } \\
\text { window size }\end{array}$ & 1,023 \\
\hline aSlotTime & Slot time duration & $20 \mu \mathrm{s}$ \\
\hline$T_{\text {SIFS }}$ & $\begin{array}{l}\text { Short interframe space } \\
\text { (aSIFSTime) }\end{array}$ & $10 \mu \mathrm{s}$ \\
\hline$T_{S Y M}$ & $\begin{array}{l}\text { Symbol interval } \\
\text { (BPSK-OFDM) }\end{array}$ & $4 \mu \mathrm{s}$ \\
\hline$T_{S I G}$ & $\begin{array}{l}\text { Signal BPSK-OFDM } \\
\text { symbol duration }\end{array}$ & $4 \mu \mathrm{s}$ \\
\hline$T_{\text {SIGEX }}$ & Signal extension duration & $6 \mu \mathrm{s}$ \\
\hline$T_{\text {PRE }}$ & $\begin{array}{l}\text { PLCP preamble } \\
\text { duration }\end{array}$ & $16 \mu \mathrm{s}$ \\
\hline$T_{\text {ACK }}$ & ACK transmission duration & $34 \mu \mathrm{s}$ \\
\hline$N_{D B P S}$ & $\begin{array}{l}\text { Number of bits per } \\
\text { OFDM symbol }\end{array}$ & 96 bits \\
\hline$M A C_{\text {header }}$ & MAC header size with QoS & 26 bytes \\
\hline
\end{tabular}

Table 4 Real-time message streams definition

\begin{tabular}{cllll}
\hline $\begin{array}{c}\text { Message } \\
\text { stream }\end{array}$ & $\begin{array}{l}\text { Mesh } \\
\text { source }\end{array}$ & $\begin{array}{l}\text { Mesh } \\
\text { destination }\end{array}$ & $\begin{array}{l}\text { Message } \\
\text { size (bytes) }\end{array}$ & $\begin{array}{l}\text { Periodicity } \\
\text { (ms) }\end{array}$ \\
\hline 1 & STA 1 & STA 25 & 80 & 50 \\
2 & STA 21 & STA 5 & 80 & 200 \\
3 & STA 25 & STA 1 & 300 & 200 \\
4 & STA 5 & STA 21 & 300 & 50 \\
\hline
\end{tabular}

being one server and four clients. In order to provide an interference equally distributed across the mesh network, the interfering nodes were selected from the middle of the mesh grid, as illustrated in Figure 1. The HTTP server is the mesh STA 13, and the HTTP clients are mesh STAs 7, 17,9 , and 19.

The simulation experiments were run in simulation batches with a duration of $400 \mathrm{~s}$, being the first $200 \mathrm{~s}$ considered for the mesh discovery process. The path selection is performed by the HWMP in proactive mode, where a mesh STA is selected as root in order to coordinate the path selection in the network.

\subsection{Network utilization model}

To determine the network load caused by the HTTP interference traffic, we adapted the utilization model presented in [46]. The network utilization $(U)$ corresponds to the intervals where the wireless channel is occupied by the transmission of MAC service data units (MSDUs) of interfering stations. Let $C=\left\{c_{1}, c_{2}, \ldots, c_{m}\right\}$ denotes the set of channel occupancy intervals of MSDUs transmission and $P=\left\{p_{1}, p_{2}, \ldots, p_{m}\right\}$ denotes the set of corresponding periodicities, where $m$ is the number of transmitted MSDUs. $U$ can be computed as follows:

$$
U=\sum_{i=1}^{m}\left(\frac{c_{i}}{p_{i}}\right)
$$

where $c_{i}$ and $p_{i}$ represent the channel occupancy of a single $\operatorname{MSDU}_{(i)}$ transmission and its corresponding periodicity, respectively. It is important to note that $p_{i}$ is the periodicity value generated by the Poisson process that models the client request interval.

Considering the previously presented HTTP model, $c_{i}$ must consider the channel occupancy intervals where clients send requests to server and it sends responses to clients. Thus, $c_{i}$ can be defined as follows:

$$
c_{i}=c_{i_{(\text {client })}}+c_{i_{(\text {server })}} \text {, }
$$

where $c_{i_{\text {(client) }}}$ and $c_{i_{\text {(server) }}}$ are the channel occupancy intervals of MSDUs transmitted by a client and by the server, respectively, both defined as follows:

$$
\begin{array}{r}
c_{i_{\text {(client) }}}=T_{\mathrm{DATA}_{(i)} \text { (client) }}+T_{\mathrm{SIFS}}+T_{\mathrm{ACK}}, \\
c_{i_{\text {(server })}}=N_{\mathrm{obj}} \times\left(T_{\mathrm{DATA}_{(i)_{(\text {server })}}+}+T_{\mathrm{SIFS}}+T_{\mathrm{ACK}}\right),
\end{array}
$$

where $T_{\text {DATA }_{(i)} \text { (client) }}$ is the transmission time of a client request, $N_{\text {obj }}$ is the number of objects sent in a server response, and $T_{\mathrm{DATA}_{(i)}(\text { server) }}$ is the transmission time of an 
object (response) sent by the server. From the IEEE 802.11 standard [4], $T_{\text {DATA }_{(i)}}$ is given by:

$$
\begin{aligned}
T_{\mathrm{DATA}_{(i)}}= & T_{\mathrm{PRE}}+T_{\mathrm{SIG}}+T_{\mathrm{SIGEX}}+T_{\mathrm{SYM}} \\
& \times\left\lceil\frac{16+6+8 \times\left(\mathrm{MSDU}_{(i)}+\mathrm{MAC}_{\text {header }}\right)}{N_{\mathrm{DBPS}}}\right\rceil .
\end{aligned}
$$

Based on the probability distributions that define the client request size and server response object size (Table 2), the MSDU size (plus headers from upper layers) was considered as the mean value of that distributions.

We defined different values for the network utilization imposed by the interfering stations, namely: $10 \%, 30 \%$, and $50 \%$. Based on the above equations, for $U=10 \%$, the client requests are sent with a periodicity of $125 \mathrm{~ms}$, for $U=30 \%$ with $42 \mathrm{~ms}$ and for $U=50 \%$ with $25 \mathrm{~ms}$. These periodicity values are used as the mean value for the Poisson process that defines the client request interval.

\subsection{Performance metrics}

As performance metrics, we considered the end-to-end delay and the average ratios of deadline misses and message losses.

\subsubsection{End-to-end delay}

The end-to-end delay is of critical importance for RT applications. If a RT message is delayed over its deadline, this message may be considered as being effectively lost. In this assessment, the end-to-end delay $\left(\delta_{e 2 e}\right)$ considers all the delays of each sender/receiver $(s / r)$ node pair until reaching the destination. The delay of each $s / r$ node pair in the multi-hop path $\left(\delta_{s / r}\right)$ is the time interval between the time instant when the acknowledge frame of a message $i$ arrives at the receiver's queue $\left(t_{r_{i}}\right)$ and the time instant when the message $i$ arrives at sender's queue $\left(t_{s_{i}}\right)$. This end-to-end delay calculation includes the processing, queuing, access, and transmission delays and is computed as follows:

$$
\begin{array}{r}
\delta_{e 2 e}=\sum_{j=1}^{N_{p}} \delta_{s / r_{(j)}}, \\
\delta_{s / r_{(j)}}=\frac{1}{N_{m}} \times \sum_{i=1}^{N_{m}}\left(t_{r_{i}}-t_{s_{i}}\right),
\end{array}
$$

where $N_{m}$ is the number of successfully received messages and $N_{p}$ is the number of $s / r$ pair nodes.

Summing up, the end-to-end delay is the required time interval to transfer a frame, measured from the moment it joins the sender's queue to the end of the frame transmission at the receiving station.

\subsubsection{Deadline miss ratio}

The deadline miss highlights the ratio of messages that exceed their bounded delivery time. In this assessment, the deadline miss ratio $(\lambda)$ is measured considering the difference between the time instant when a message $i$ was received at the destination and the time instant when message $i$ was sent from the source. If the difference between these time instants is greater than the message periodicity (i.e., its deadline), the message is deemed to have missed its deadline. In addition, a message that is dropped (due to exceeding its transmission attempt count or due to the queue control algorithm) is also deemed to have exceeded its deadline. Thus, the deadline miss ratio is directly affected by the message loss ratio. This ratio is computed as follows:

$$
\lambda=\frac{m_{\text {missed }}+m_{\mathrm{dropped}}}{m_{\mathrm{sent}}},
$$

where $m_{\text {missed }}$ is the total number of successfully received messages that missed their deadline, $m_{\text {dropped }}$ is the total number of undelivered messages (that obviously also missed their deadlines), and $m_{\text {sent }}$ is the total number of sent messages.

\subsubsection{Message loss ratio}

The message loss ratio is defined for a receiving station as the number of dropped messages during a transmission. In this assessment, the message loss ratio $(\sigma)$ is measured considering the messages that were effectively dropped due to transmission error or due to exceeding the transmission attempt count. It can be computed as follows:

$$
\sigma=\frac{m_{\mathrm{dropped}}}{m_{\mathrm{sent}}}
$$

where $m_{\text {dropped }}$ is the total number of dropped messages and $m_{\text {sent }}$ is the total number of sent messages.

\section{Simulation results}

Different communication scenarios have been simulated to assess the behavior of RT traffic when the wireless channel is shared with non-RT traffic generated by a set of interfering stations. For the sake of simplicity, only the results that concern RT traffic will be presented.

\subsection{Impact of external traffic sources over the end-to-end delay}

The first simulation scenario concerns the assessment of the end-to-end delay of four RT message streams, as defined in Table 4, when the overall network utilization is increased by a set of interfering stations.

Figure 2 illustrates the histogram and the cumulative distribution function (CDF) of end-to-end delay of RT 


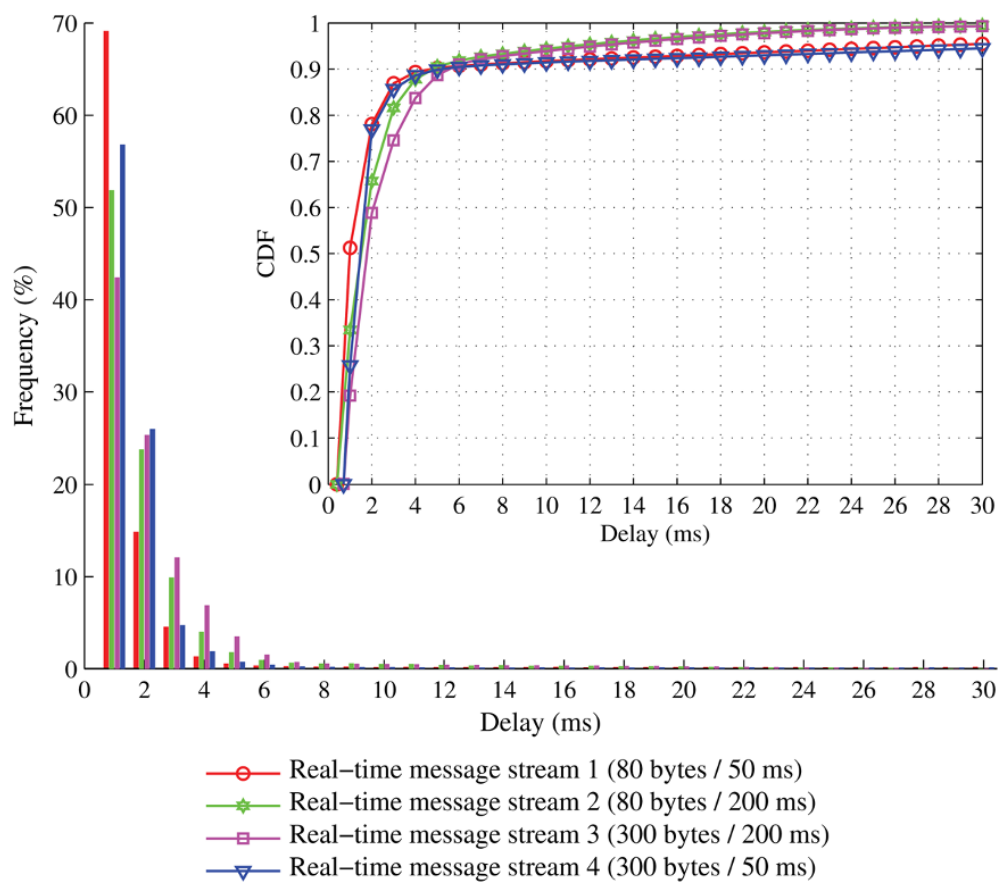

Figure 2 Histogram and CDF of end-to-end delay of RT message streams without any external traffic sources.
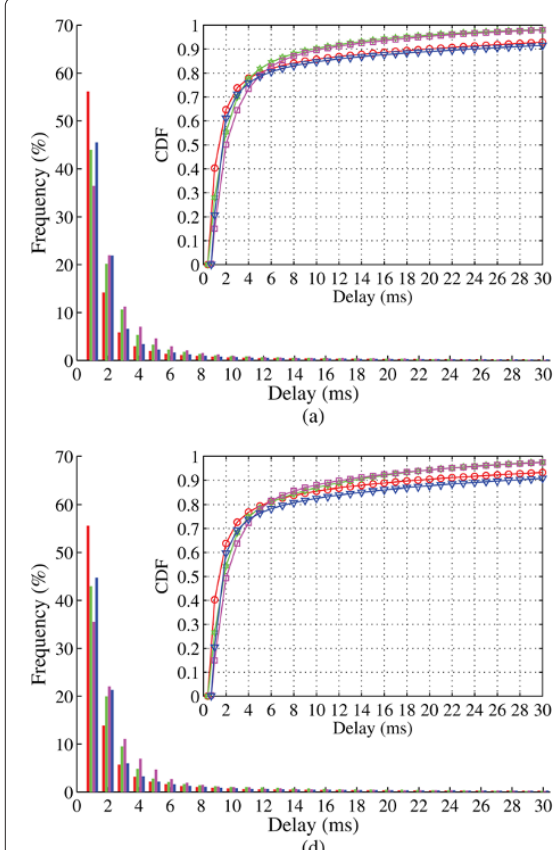

(d)

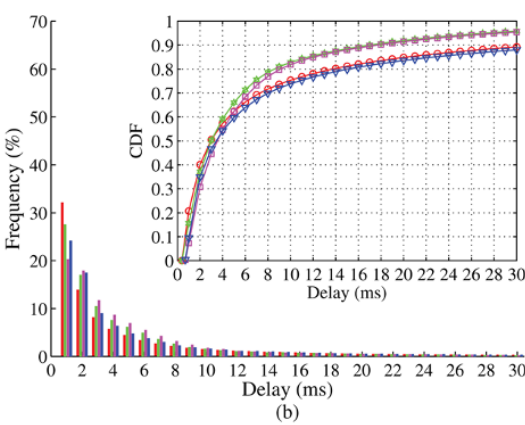

(b)

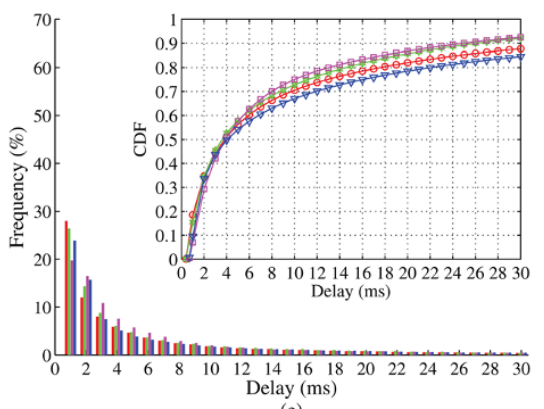

(e)

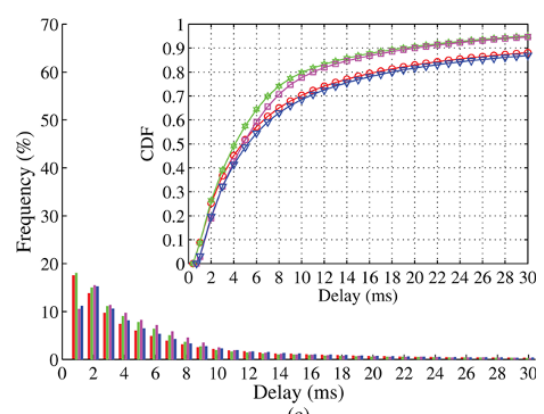

(c)

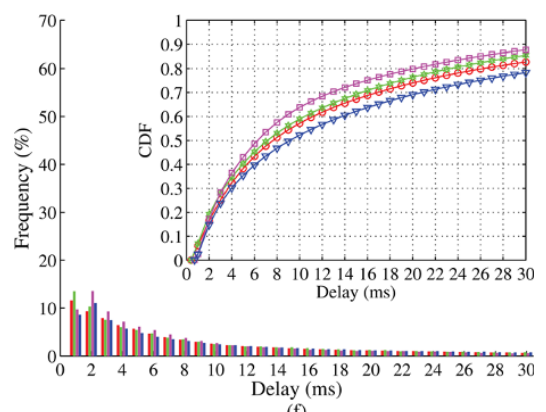

(f)

$\rightarrow$ Real-time message stream 1 ( 80 bytes $/ 50 \mathrm{~ms}) \rightarrow$ Real-time message stream 3 ( 300 bytes $/ 200 \mathrm{~ms})$

Figure 3 Histograms and CDFs of end-to-end delay of RT message streams with external traffic sources. (a) $10 \%$, (b) $30 \%$, and (c) $50 \%$ of network utilization with maximum priority traffic separation between RT traffic and non-RT traffic; (d) $10 \%$, (e) $30 \%$, and (f) 50\% of network utilization with mixed priority traffic separation between RT traffic and non-RT traffic. 
message streams without any interfering traffic sources in the network, where it can be observed the default behavior of RT communication.

Based on Figure 2, it is possible to notice that RT message streams with the lowest periodicity experiment slightly increased delay, regardless of the message size. This behavior is due to the number of messages that are sent in a shorter period, which are prone to higher delays since the network needs to deal with other traffic and the wireless channel is not always available for such shorter interval requests. However, the difference between the end-to-end delay of the different message streams is negligible.

Regarding the impact of non-RT traffic over the RT communication, Figure 3(a),(b),(c) presents the histograms and CDFs of end-to-end communication delay with maximum priority traffic separation between RT traffic and non-RT traffic (as defined in Section 4) and
Figure 3(d),(e),(f) presents the histograms and CDFs of end-to-end communication delay with mixed priority traffic separation between RT traffic and non-RT traffic (also as defined in Section 4).

According to the results, it is noticeable that the end-toend delay of RT traffic increases as the overall network utilization increases. These results clearly show the impact of the interference traffic upon the RT traffic behavior, even for the case when the maximum priority traffic separation is imposed. With a network load equal or greater than $30 \%$, it is clear that the end-to-end delay of all RT message streams is highly affected. For the case when the mixed priority traffic separation is imposed, the delay is even more affected when compared to the maximum priority traffic separation. This set of simulations indicates that the mandatory EDCA MAC mechanism, defined in the IEEE 802.11s, is not able to impose the traffic separation required for $\mathrm{RT}$ communication when using the default

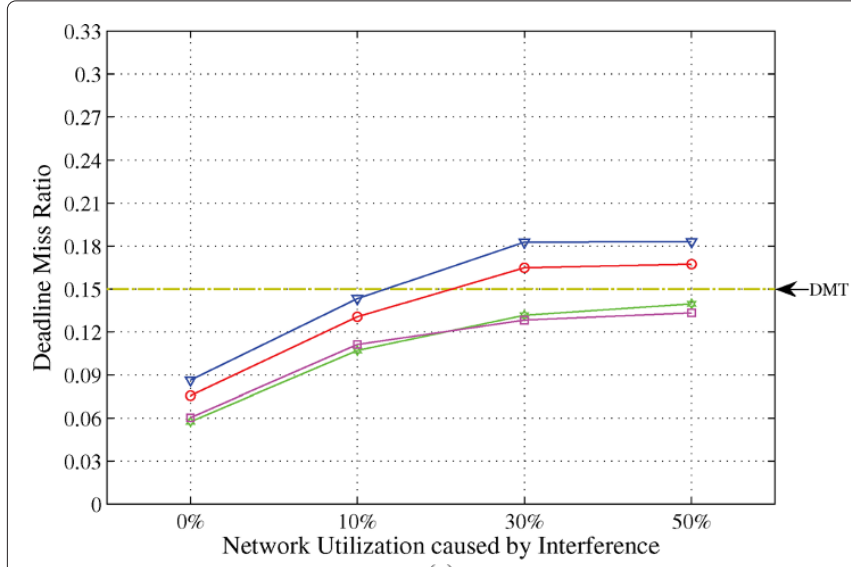

(a)

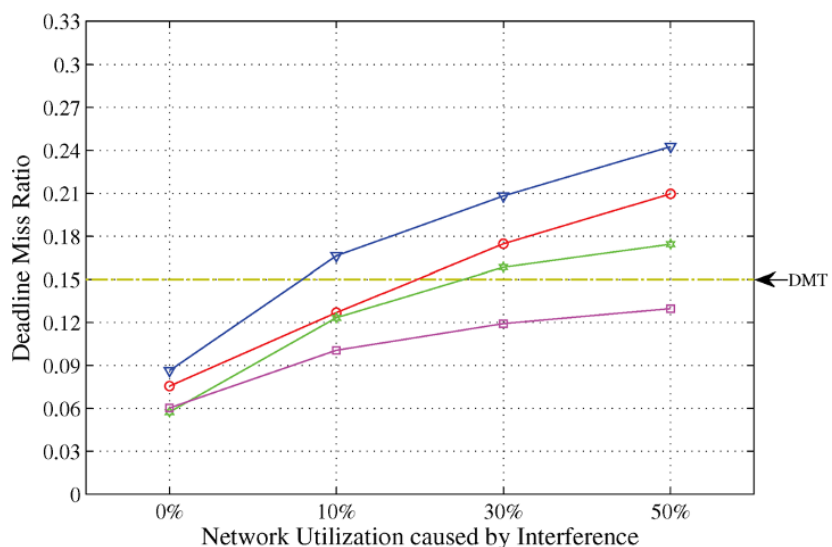

(c)

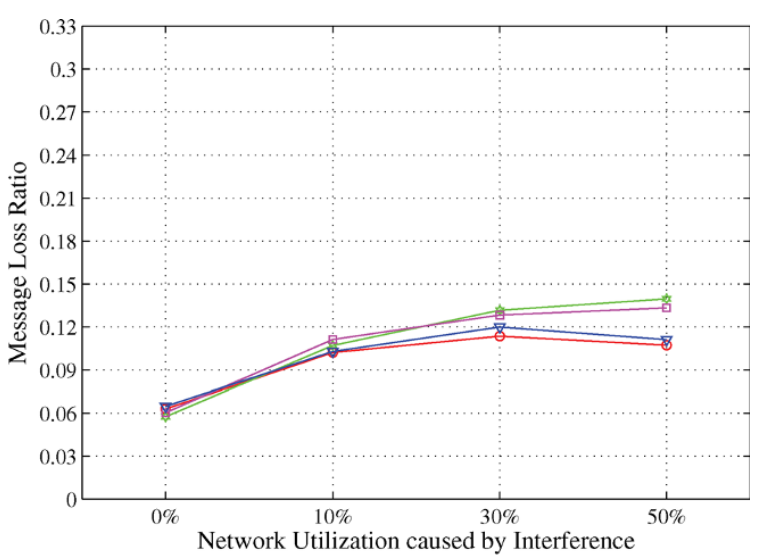

(b)

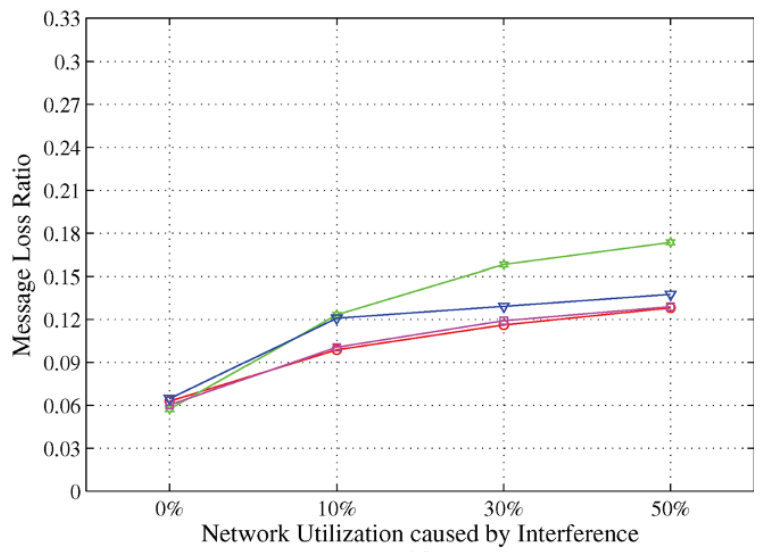

(d)

$\square$ - Real-time message stream 3 (300 bytes / $200 \mathrm{~ms}$ )

$\rightarrow$ Real-time message stream 4 (300 bytes / $50 \mathrm{~ms}$ )

Figure 4 Average ratios of deadline misses and message losses of RT message streams. (a) deadline miss ratio and (b) message loss ratio with maximum priority traffic separation between RT traffic and non-RT traffic; (c) deadline miss ratio and (d) message loss ratio with mixed priority traffic separation between $\mathrm{RT}$ traffic and non-RT traffic. 
Table 5 Effects of varying aCW $\mathrm{CW}_{\min }$ and $\mathrm{aCW}_{\max }$ upon $\mathrm{RT}$ message streams 1 and 2

\begin{tabular}{|c|c|c|c|c|c|c|c|}
\hline \multirow{2}{*}{$\mathrm{aCW}_{\min }$} & \multirow{2}{*}{$\mathrm{aCW}_{\max }$} & \multicolumn{2}{|c|}{ Average end-to-end delay (ms) } & \multicolumn{2}{|c|}{ Deadline miss ratio (\%) } & \multicolumn{2}{|c|}{ Message loss ratio (\%) } \\
\hline & & Stream 1 & Stream 2 & Stream 1 & Stream 2 & Stream 1 & Stream 2 \\
\hline 1 & 3 & 11.27 & 8.36 & 16.2 & 12.5 & 10.1 & 12.5 \\
\hline 1 & 7 & 12.15 & 8.76 & 16.7 & 12.3 & 10.1 & 12.3 \\
\hline 1 & 15 & 12.47 & 9.03 & 16.8 & 12.8 & 10.1 & 12.7 \\
\hline$\vdots$ & $\vdots$ & & & & & & \\
\hline 1 & 1,023 & 13.62 & 9.28 & 16.7 & 13.1 & 9.5 & 13.0 \\
\hline 3 & 7 & 13.20 & 9.36 & 16.6 & 12.1 & 9.8 & 12.1 \\
\hline 3 & 15 & 13.36 & 9.59 & 16.4 & 12.2 & 9.6 & 12.2 \\
\hline 3 & 31 & 13.90 & 9.74 & 17.0 & 12.3 & 10.0 & 12.3 \\
\hline$\vdots$ & $\vdots$ & & & & & & \\
\hline 3 & 1,023 & 13.03 & 9.79 & 15.9 & 12.3 & 9.5 & 12.3 \\
\hline 7 & 15 & 14.30 & 11.02 & 16.7 & 12.2 & 9.9 & 12.1 \\
\hline 7 & 31 & 16.68 & 11.68 & 17.5 & 11.8 & 9.6 & 11.7 \\
\hline 7 & 63 & 16.31 & 11.70 & 17.3 & 12.0 & 9.5 & 11.9 \\
\hline$\vdots$ & & & & & & & \\
\hline 7 & 1,023 & 16.84 & 12.72 & 17.7 & 12.8 & 9.8 & 12.6 \\
\hline 15 & 31 & 21.92 & 14.79 & 19.9 & 12.6 & 9.9 & 12.4 \\
\hline$\vdots$ & & & & & & & \\
\hline 15 & 1,023 & 24.08 & 15.42 & 20.6 & 12.5 & 9.7 & 12.2 \\
\hline$\vdots$ & $\vdots$ & & & & & & \\
\hline 255 & 1,023 & 220.41 & 109.79 & 63.2 & 26.2 & 11.7 & 12.8 \\
\hline 511 & 1,023 & 542.52 & 279.33 & 83.7 & 50.2 & 14.5 & 14.2 \\
\hline
\end{tabular}

The italicized values represent the RT communication performance when using EDCA voice class, i.e., $\mathrm{aCW}_{\min }=3$ and $\mathrm{aCW} \mathrm{max}_{\max }=7$.

Table 6 Effects of varying $\mathrm{aCW}_{\min }$ and $\mathrm{aCW}_{\max }$ upon $\mathrm{RT}$ message streams 3 and 4

\begin{tabular}{|c|c|c|c|c|c|c|c|}
\hline \multirow{2}{*}{$\mathrm{aCW}_{\min }$} & \multirow{2}{*}{$\mathrm{aCW}_{\max }$} & \multicolumn{2}{|c|}{ Average end-to-end delay (ms) } & \multicolumn{2}{|c|}{ Deadline miss ratio (\%) } & \multicolumn{2}{|c|}{ Message loss ratio (\%) } \\
\hline & & Stream 3 & Stream 4 & Stream 3 & Stream 4 & Stream 3 & Stream 4 \\
\hline 1 & 3 & 7.34 & 15.17 & 10.9 & 20.0 & 10.9 & 12.4 \\
\hline 1 & 7 & 7.87 & 15.96 & 11.0 & 19.8 & 10.9 & 11.7 \\
\hline 1 & 15 & 8.15 & 17.07 & 10.5 & 20.9 & 10.5 & 11.9 \\
\hline & $\vdots$ & & & & & & \\
\hline 1 & 1,023 & 8.13 & 18.11 & 11.2 & 21.2 & 11.1 & 12.4 \\
\hline 3 & 7 & 8.02 & 16.70 & 11.0 & 20.4 & 11.0 & 12.2 \\
\hline 3 & 15 & 8.72 & 16.91 & 10.9 & 20.0 & 10.9 & 11.7 \\
\hline 3 & 31 & 8.88 & 16.65 & 11.0 & 20.1 & 10.9 & 11.9 \\
\hline$\vdots$ & $\vdots$ & & & & & & \\
\hline 3 & 1,023 & 8.30 & 16.86 & 10.5 & 20.5 & 10.5 & 12.1 \\
\hline 7 & 15 & 9.79 & 18.01 & 10.4 & 20.6 & 10.4 & 11.8 \\
\hline 7 & 31 & 10.25 & 19.21 & 11.5 & 21.6 & 11.4 & 12.4 \\
\hline 7 & 63 & 10.55 & 19.59 & 11.0 & 21.3 & 11.0 & 11.9 \\
\hline$\vdots$ & & & & & & & \\
\hline 7 & 1,023 & 10.76 & 20.03 & 11.3 & 21.8 & 11.1 & 12.4 \\
\hline 15 & 31 & 13.59 & 24.33 & 10.9 & 23.2 & 10.7 & 11.7 \\
\hline : & & & & & & & \\
\hline 15 & 1,023 & 14.56 & 26.03 & 10.9 & 23.2 & 10.6 & 11.2 \\
\hline$\vdots$ & & & & & & & \\
\hline 255 & 1,023 & 106.46 & 215.28 & 24.5 & 63.4 & 11.2 & 13.9 \\
\hline 511 & 1,023 & 283.65 & 527.28 & 48.7 & 84.0 & 11.7 & 16.5 \\
\hline
\end{tabular}


Table 7 HWMP default parameters

\begin{tabular}{ll}
\hline Parameter & Value \\
\hline Path lifetime & $5 \mathrm{~s}$ \\
Traversal time & $500 \mathrm{~ms}$ \\
\hline
\end{tabular}

set of parameters, since the non-RT interference traffic (background) affects the RT communication (voice), as the dispersion of the histograms related to a utilization of $30 \%$ to $50 \%$ is considerably higher.

\subsection{Impact of external traffic sources over the average ratios of deadline misses and message losses}

The second simulation scenario concerns the assessment of average ratios of deadline misses and message losses for the RT message streams. Figure 4 illustrates the impact of interference traffic over these metrics.

The deadline miss ratio is directly influenced by both the message loss ratio (Figure $4(\mathrm{~b}),(\mathrm{d})$ ) and the excessive access delay of RT message streams (Figure 3). A noticeable increase of deadline misses can be observed, caused by the increasing delay as the network utilization increases.

From the above results, it is possible to notice that maximum priority traffic separation presents less deadline misses when compared to the mixed priority traffic separation. In the mixed priority separation scenario, the traffic transmitted at video and voice classes severely impact the deadline miss ratio. Also, message streams with longer periodicity values tend to present less deadline misses, regardless of their size, since they contend for the medium access less frequently. On the other hand, message streams with shorter periodicity values tend to present higher number of deadline misses since there are more messages being transmitted in a shorter time interval, which are therefore more prone to losses.

Considering message streams 1 and 4, which are the streams with shorter periodicity values (Table 4), they clearly exceed a DMT value of $15 \%$ (target defined in Section 4) when the network utilization is greater than approximately $12 \%$ and $21 \%$, respectively, even for the case when the maximum priority traffic separation is imposed. This behavior worsens when the mixed priority traffic separation is imposed, where the DMT is exceeded when the network utilization is greater than approximately $8 \%$ and $19 \%$, respectively.

Considering message streams 2 and 3, which are the streams with longer periodicity values, they do not exceed the DMT value with the maximum priority traffic separation when the network utilization is less than or equal to $50 \%$. Also, it is important to note that deadline misses are due to message losses, as can be seen in the Figure 4(a),(b). This means that all successfully transmitted messages arrived on time at the destination. However, for the mixed priority traffic separation scenario, the message stream 2 slightly exceeds the DMT, for the case where the network utilization is greater than approximately $26 \%$. Once again, the deadline misses of message stream 2 are caused by message losses, as can be extracted from Figure 4(c),(d). This result indicates that streams with longer periodicity values suffer less impact when compared with streams with shorter periodicity values, where deadline misses are caused essentially by message losses.

The results suggest that RT communication performance is highly dependent on the traffic type and on the imposed network utilization. Also, the periodicity values of message streams play an important role in the RT communication performance, regardless of messages size. Thus, the IEEE 802.11s standard with the mandatory EDCA scheme may only reliably support RT traffic with shorter periodicity values when the network utilization is below $20 \%$ and the interference traffic occurs at lower priority classes. However, when high priority classes are used to transmit interference traffic, the RT communication is severely impacted by the network utilization, since EDCA is not able to provide an acceptable service differentiation.

\subsection{Tuning EDCA and HWMP parameters}

Considering that we have full control upon the parameters of RT stations, it is reasonable to propose the selection of adequate parameters to improve the RT message streams transmission. Therefore, in this third scenario, we make a sensitivity analysis to assess the effects of varying EDCA contention and HWMP routing parameters upon the RT communication behavior. The set of parameters that are subject to analysis are: the CW size of the EDCA mechanism and the traversal time and path lifetime of the HWMP.

\section{Table 8 RT communication performance with different values for traversal time}

\begin{tabular}{|c|c|c|c|c|c|c|c|c|}
\hline & \multicolumn{8}{|c|}{ Traversal time (ms) } \\
\hline & 100 & 1,000 & 100 & 1,000 & 100 & 1,000 & 100 & 1,000 \\
\hline & \multicolumn{2}{|c|}{ Stream 1} & \multicolumn{2}{|c|}{ Stream 2} & \multicolumn{2}{|c|}{ Stream 3} & \multicolumn{2}{|c|}{ Stream 4} \\
\hline Average end-to-end delay (ms) & 10.11 & 14.43 & 6.97 & 9.76 & 7.29 & 9.02 & 10.35 & 17.12 \\
\hline Deadline miss ratio (\%) & 13.6 & 17.6 & 10.6 & 15.0 & 9.4 & 12.2 & 14.8 & 20.7 \\
\hline Message loss ratio (\%) & 9.6 & 12.4 & 10.6 & 15.0 & 9.4 & 12.2 & 10.7 & 13.8 \\
\hline
\end{tabular}


Table 9 RT communication performance with different values for path lifetime

\begin{tabular}{|c|c|c|c|c|c|c|c|c|}
\hline & \multicolumn{8}{|c|}{ Path lifetime (s) } \\
\hline & 1 & 10 & 1 & 10 & 1 & 10 & 1 & 10 \\
\hline & \multicolumn{2}{|c|}{ Stream 1} & \multicolumn{2}{|c|}{ Stream 2} & \multicolumn{2}{|c|}{ Stream 3} & \multicolumn{2}{|c|}{ Stream 4} \\
\hline Average end-to-end delay (ms) & 34.27 & 8.54 & 10.99 & 6.66 & 12.89 & 6.57 & 40.44 & 9.72 \\
\hline Deadline miss ratio (\%) & 50.9 & 9.7 & 46.6 & 9.0 & 42.6 & 6.7 & 49.9 & 12.7 \\
\hline Message loss ratio (\%) & 33.3 & 6.8 & 46.5 & 9.0 & 42.5 & 6.7 & 29.4 & 9.0 \\
\hline
\end{tabular}

\subsubsection{Varying the contention window size}

As seen in the previous section, the EDCA mechanism presents a poor performance to support RT traffic when the wireless channel is shared with interfering traffic sources, notably when the overall network utilization is above $20 \%$. In order to investigate the impact of EDCA parameters in the WMNs communication behavior, we assess the effects of varying the contention window size used to transmit RT traffic. Similarly to the assessment presented in [17], we examined all the possible combinations of $\mathrm{aCW}_{\min }$ and $\mathrm{aCW}$ max between 1 and 1,023. We chose to reproduce the scenario where the network utilization is $30 \%$ and the mixed priority traffic separation is imposed between RT traffic and interference traffic. Tables 5 and 6 present the main results for real-time message streams 1 and 2 and for streams 3 and 4, respectively. For the sake of presentation, we highlighted the results for the EDCA voice class.

Contrarily to the results observed in [17], where it is highlighted a performance improvement with larger $\mathrm{CW}$ values (e.g., $\mathrm{aCW} \min =15$ and $\mathrm{aCW}_{\max }=31$ ) over the default voice class (i.e., $\mathrm{aCW}_{\min }=3$ and $\mathrm{aCW}_{\max }=7$ ), in this mesh scenario smaller CW values (e.g., $\mathrm{aCW}_{\min }=1$ and $\mathrm{aCW}_{\max }=3$ ) present better performance over the voice class. It is important to note the assessment presented in [17] considers a single-hop ad hoc network with no hidden terminals. In this assessment, due to the multi-hop communication and grid topology, there are several hidden terminals in the WMN. However, the interference caused by a transmitting station affects only the stations in the related coverage area, i.e., other message streams can be simultaneously transmitted in another network area. Consequently, there is a performance degradation of RT communication as the size of $\mathrm{CW}$ increases. Messages with higher $\mathrm{CW}$ values spend more time in EDCA queues, since they backoff for longer time intervals and consequently the deadline miss ratio increases.

\subsubsection{Varying HWMP parameters}

The HWMP routing protocol has a set of protocol parameters that are essential for the path management and directly impacts on the communication performance in the WMN. One of those parameters is the traversal time (defined by the attribute dot11MeshHWMPnetDiameterTraversalTime), an upper bound for the time interval to propagate an HWMP element across the mesh network. Basically, whenever a frame takes a time interval to traverse the mesh that is longer than the traversal time, it is discarded and no longer delivered at its destination.

Another HWMP parameter is the path lifetime (composed of the attributes dot11MeshHWMPactivePathToRootTimeout and dot11MeshHWMPactivePathTimeout), which is a parameter that specifies the time during which a mesh STA shall consider the forwarding information to the root mesh STA to be valid (proactive mode) and to any other mesh STA (reactive mode), respectively. Basically, the path lifetime defines an upper bound for the path duration; whenever it expires, the path is reset and a new path discover procedure is initiated.

These two HWMP parameters are the most relevant when considering the RT communication behavior in a WMN. Therefore, the sensitivity analysis performed in this paper addresses specifically these two network parameters and its effects over the communication performance. Once again, it was considered a communication scenario with a network utilization of $30 \%$ and a mixed priority traffic separation between the real-time traffic and the interfering HTTP traffic.

The default values of HWMP parameters are defined in Table 7 and the communication performance with these values can be observed in Tables 5 and 6 for default EDCA voice class parameters, i.e., $\mathrm{aCW}_{\min }=3$ and $\mathrm{aCW}_{\max }=$ 7. For the sake of simplicity, these values will not be repeated in Tables 8, 9, and 10, which present the communication performance when the default values of HWMP parameters are changed.

Table 10 RT communication performance with best contention and routing parameter values

\begin{tabular}{lcccc}
\hline & $\begin{array}{c}\text { Stream } \\
\mathbf{1}\end{array}$ & $\begin{array}{c}\text { Stream } \\
\mathbf{2}\end{array}$ & $\begin{array}{c}\text { Stream } \\
\mathbf{3}\end{array}$ & $\begin{array}{c}\text { Stream } \\
\mathbf{4}\end{array}$ \\
\hline $\begin{array}{l}\text { Average end-to-end } \\
\text { delay (ms) }\end{array}$ & 6.80 & 5.30 & 5.44 & 8.35 \\
Deadline miss ratio (\%) & 8.20 & 7.05 & 6.13 & 9.59 \\
Message loss ratio (\%) & 5.49 & 7.03 & 6.11 & 6.26 \\
\hline
\end{tabular}


When varying the traversal time value (see Table 8 ), it is possible to notice an improvement in the communication performance. For example, considering the message stream 4 , the message loss ratio decreases from $12.2 \%$ (when using the default traversal time value) to $10.7 \%$ (when decreasing the traversal time value to $100 \mathrm{~ms}$ ). The opposite occurs when the traversal time value is increased to $1,000 \mathrm{~ms}$. Therefore, to optimize the RT communication behavior it is recommended to set the traversal time to the maximum message stream period value, i.e., $200 \mathrm{~ms}$ for this communication scenario.

Table 9 presents the results of varying the path lifetime value from $5 \mathrm{~s}$ to $1 \mathrm{~s}$ and $10 \mathrm{~s}$. When the path lifetime value is lower than the default value, it is possible to notice an improvement in the RT communication performance. This is an expected result, since to reliably support real-time traffic, the path forwarding information should stay valid as long as possible. Otherwise, new path discovery procedures would be frequently performed, which degrades the RT communication performance. Therefore, contrarily to the commonly used HTTP applications, for RT traffic transmission, it is desirable to establish and maintain the forwarding paths valid as long as possible.

Based on the best CW, traversal time and path lifetime values extracted from the previous simulation scenario, we repeated the simulation from the third scenario. Table 10 illustrates the performance metrics when considering this scenario with $\mathrm{aCW}_{\min }=1$ and $\mathrm{aCW}_{\max }=3$, traversal time $=200 \mathrm{~ms}$ and path lifetime $=10 \mathrm{~s}$. As a result of this sensitivity analysis, we may conclude that by carefully setting the $\mathrm{CW}$, traversal time and the path lifetime parameters we may significantly increase the quality of service provided to the RT traffic supported by the WMN.

\section{Conclusions}

This paper presents a simulation assessment of the impact of non-real-time (non-RT) traffic sources upon RT communication in IEEE 802.11s WMNs. A set of simulation experiments have been conducted in ns-3, to assess the RT communication behavior when the wireless channel is shared with external interferences.

From the simulation results, it can be observed that the RT communication behavior is directly affected by the presence of non-RT traffic sources. The default parameter values used for EDCA are not adequate to provide the required service differentiation, as the EDCA mechanism is not able to separate the higher priority traffic from the traffic transmitted at lower priority classes. When increasing the network utilization, it can be observed a degradation of the performance metrics, indicating that the default parameter values should be set differently.
Therefore, a sensitivity analysis has been performed to evaluate the impact of some EDCA/HWMP key parameters upon the RT communication behavior. By carefully setting the EDCA CW size and the HWMP traversal time and path lifetime parameters, it was possible to enhance the RT communication behavior and consequently to improve the support of RT communication in WMNs. Basically, it could be observed that the RT communication behavior is significantly improved from the usage of: a) smaller CW sizes for the EDCA mechanism, b) larger path lifetime values for the HWMP protocol, and c) mesh traversal times adjusted to the longest periodicity value in use for the supported RT message streams.

\section{Competing interests}

The authors declare that they have no competing interests.

\section{Acknowledgements}

This work was partially funded by the Instituto de Engenharia Mecânica e Gestão Industrial (IdMEC/INEGI - FEUP), by the Fundação para a Ciência e a Tecnologia - FCT (POPH/QREN/PORTUGAL/FSE) under the reference SFRH/BD/70077/2010 and by the Conselho Nacional de Desenvolvimento Científico e Tecnológico - CNPq - Brazil (400508/2014-1).

\section{Author details}

${ }^{1}$ IdMEC/INEGI/INESC-Tec - Faculdade de Engenharia da Universidade do Porto, Rua Dr. Roberto Frias, s/n, 4200-465 Porto, Portugal. ${ }^{2}$ Universidade Federal de Santa Catarina, Rua Pedro João Pereira, 150, 88900-000 Araranguá-SC, Brazil.

Received: 4 April 2014 Accepted: 17 November 2014

Published: 11 December 2014

\section{References}

1. A Sgora, D Vergados, P Chatzimisios, in Mobile Lightweight Wireless Systems, vol. 13, ed. by F Granelli. IEEE 802.11s wireless mesh networks: challenges and perspectives (Springer Berlin, 2009), pp. 263-271. doi:10.1007/978-3-642-03819-8_25

2. RC Carrano, LCS Magalhães, DCM Saade, CVN Albuquerque, IEEE 802.11s multihop MAC: a tutorial. IEEE Commun. Surv. Tutor. 13(1), 52-67 (2011). doi:10.1109/SURV.2011.040210.00037

3. K Mase, Layer 3 wireless mesh networks: mobility management issues. IEEE Commun. Mag. 49(7), 156-163 (2011). doi:10.1109/MCOM.2011.5936169

4. IEEE Standard for Information Technology - Telecommunications and information exchange between systems local and metropolitan area networks - specific requirements part 11: wireless LAN medium access control (MAC) and physical layer (PHY) specifications. IEEE Std 802.11-2012 (Revision of IEEE Std 802.11-2007), 1-2793 (2012). doi:10.1109/IEEESTD.2012.6178212

5. IF Akyildiz, X Wang, W Wang, Wireless mesh networks: a survey. Comput. Netw. 47(4)، 445-487 (2005). doi:10.1016/j.comnet.2004.12.001

6. A Hamidian, U Körner, in 21st IEEE International Teletraffic Congress. Distributed reservation-based QOS in ad hoc networks with internet access connectivity (France Paris, 2009), pp. 1-8

7. GR Hiertz, J Habetha, P May, E Weib, R Bagul, S Mangold, in 14th IEEE Proceedings on Personal, Indoor and Mobile Radio Communications, vol. 3. A decentralized reservation scheme for IEEE 802.11 ad hoc networks, (2003), pp. 2576-2580. doi:10.1109/PIMRC.2003.1259192

8. X Yang, Z Rosberg, Z Cao, RP Liu, in IEEE International Conference on Communications (ICC). Admission control for wireless mesh networks based on active neighbor bandwidth reservations (Cape Town, South Africa, 2010), pp. 1-6. doi:10.1109/ICC.2010.5502668

9. R Moraes, F Vasques, P Portugal, in IEEE Network Operations and Management Symposium (NOMS). A 2-tier architecture to support real-time communication in CSMA-based networks (Salvador, Bahia, Brazil, 2008), pp. 1061-1066. doi:10.1109/NOMS.2008.4575279 
10. E Carlson, C Prehofer, C Bettstetter, H Karl, A Wolisz, A distributed end-to-end reservation protocol for IEEE 802.11-based wireless mesh networks. IEEE J. Select. Areas Commun. (J-SAC) 24(11), 2018-2027 (2006). doi:10.1109/JSAC.2006.881633

11. A Krasilov, A Lyakhov, A Safonov, in IEEE 8th International Conference on Mobile Adhoc and Sensor Systems (MASS). Interference, even with MCCA channel access method in IEEE 802.11s mesh networks (Valencia, Spain, 2011), pp. 752-757. doi:10.1109/MASS.2011.83

12. M Pinheiro, F Vasques, S Sampaio, P Souto, in 6th Annual IEEE Communications Society Conference on Sensor, Mesh and Ad HoC Communications and Networks Workshops (SECON Workshops). DHT-based cluster routing protocol for IEEE802.11s mesh networks (Rome, Italy, 2009), pp. 1-6. doi:10.1109/SAHCNW.2009.5172928

13. F Nawab, K Jamshaid, B Shihada, $\mathrm{PH}$ Ho, Fair packet scheduling in wireless mesh networks. Ad Hoc Netw. 13(Part B), 414-427 (2014). doi:10.1016/j.adhoc.2013.09.002

14. WJ Jung, SH Min, BG Kim, HS Choi, JY Lee, BC Kim, in International Conference on Information Networking (ICOIN). R-HWMP. Reservation-based HWMP supporting end-to-end QoS in wireless mesh networks, (2013), pp. 385-390. doi:10.1109/ICOIN.2013.6496409

15. A Lyakhov, I Pustogarov, in Multiple Access Communications. Lecture Notes in Computer Science, vol. 7642, ed. by BB. Intra-flow interference study in IEEE 802.11s mesh networks (Springer Berlin, 2010), pp. 127-138. doi:10.1007/978-3-642-15428-7_14

16. CMD Viegas, S Sampaio, F Vasques, P Portugal, P Souto, in 9th IEEE International Workshop on Factory Communication Systems (WFCS). Assessment of the interference caused by uncontrolled traffic sources upon real-time communication in IEEE 802.11-based mesh networks, (2012), pp. 59-62. doi:10.1109/WFCS.2012.6242541

17. R Moraes, P Portugal, F Vasques, RF Custódio, Assessment of the IEEE 802.11e EDCA protocol limitations when dealing with real-time communication. EURASIP J. Wireless Commun. Netw. 2010, 1-14 (2010). doi:10.1155/2010/351480

18. R Moraes, P Portugal, F Vasques, in IEEE Conference on Emerging Technologies and Factory Automation (ETFA). Simulation analysis of the IEEE 802.11e EDCA protocol for an industrially-relevant real-time communication scenario, (2006), pp. 202-209. doi:10.1109/ETFA.2006.355207

19. CE Perkins, E Belding-Royer, S Das, Ad hoc on-demand distance vector (AODV) routing. Request for Comments (RFC) 3561, Internet Engineering Task Force (IETF) (July 2003). Available: http://www.rfc-editor.org/rfc/ rfc3561.txt [Accessed 20 May 2014]

20. M Bahr, J Wang, X Jia, in Wireless Mesh Networking: Architectures, Protocols and Standards, ed. by Y Zhang, J Luo, and H Hu. Routing in wireless mesh networks (Taylor \& Francis New York, USA, 2006), pp. 133-134

21. IEEE standard for local and metropolitan area networks: media access control (MAC) bridges. IEEE Std 802.1D-2004 (Revision of IEEE Std 802.1D-1998) 1-277 (2004). doi:10.1109/IEEESTD.2004.94569

22. D-J Deng, R-S Chang, A priority scheme for IEEE 802.11 DCF access method. IEICE Trans. Commun. (Japan) E82-B(1), 96-102 (1999)

23. J Hui, M Devetsikiotis, A unified model for the performance analysis of IEEE 802.11e EDCA. IEEE Trans. Commun. 53(9), 1498-1510 (2005). doi:10.1109/TCOMM.2005.855013

24. Z-N Kong, DHK Tsang, B Bensaou, D Gao, Performance analysis of IEEE 802.11 e contention-based channel access. IEEE J. Select. Areas Commun. 22(10), 2095-2106 (2004). doi:10.1109/JSAC.2004.836019

25. Y Xiao, Performance analysis of priority schemes for IEEE 802.11 and IEEE 802.11e wireless LANs. IEEE Trans. Wireless Commun. 4(4), 1506-1515 (2005). doi:10.1109/TWC.2005.850328

26. Z Tao, S Panwar, Throughput and delay analysis for the IEEE 802.11e enhanced distributed channel access. IEEE Trans. Commun. 54(4), 596-603 (2006). doi:10.1109/TCOMM.2006.873066

27. L Xiong, G Mao, Saturated throughput analysis of IEEE 802.11e EDCA. Comput. Netw. 51(11), 3047-3068 (2007). doi:10.1016/j.comnet.2007.01.002

28. E Karamad, F Ashtiani, Performance analysis of IEEE 802.11 DCF and 802.11e EDCA based on queueing networks. IET Commun. 3(5), 871-88 (2009). doi:10.1049/iet-com.2008.0676

29. M El Masri, S Abdellatif, in 8th IFAC International Conference on Fieldbuses and Networks in Industrial and Embedded Systems. Managing the virtual collision in IEEE 802.11e EDCA, (2009), pp. 104-109. doi:10.3182/20090 520-3-KR-3006.00016
30. Y He, J Sun, X Ma, AV Vasilakos, R Yuan, W Gong, Semi-random backoff: towards resource reservation for channel access in wireless LANs. IEEE/ACM Trans. Netw. 21(1), 204-217 (2013). doi:10.1109/TNET.2012. 2202323

31. T Sanguankotchakorn, A Gopalasingham, N Sugino, in 4th International Conference on Intelligent Systems Modelling Simulation (ISMS). Adaptive channel access mechanism for real time traffic over IEEE 802.11e Wi-Fi network, (2013), pp. 486-491. doi:10.1109/ISMS.2013.80

32. G-H Hwang, D-H Cho, Performance analysis on coexistence of EDCA and legacy DCF stations in IEEE 802.11 wireless LANs. IEEE Trans. Wireless Commun. 5(12), 3355-3359 (2006). doi:10.1109/TWC.2006.256955

33. Q Ni, Performance analysis and enhancements for IEEE 802.11e wireless networks. IEEE Network 19(4), 21-27 (2005). doi:10.1109/MNET.2005. 1470679

34. CT Calafate, P Manzoni, MR Malumbres. Assessing the effectiveness of IEEE 802.11e in multi-hop mobile network environments, (2004), pp. 205-212. doi:10.1109/MASCOT.2004.1348232

35. B Xiang, M Yu-Ming, in International Conference on Wireless Communications, Networking and Mobile Computing (WiCom). The impact of hidden nodes on MAC layer performance of multi-hop wireless networks using IEEE802.11e protocol, (2007), pp. 1479-1483. doi:10.1109/WICOM.2007.374

36. KXu, M Gerla, S Bae, Effectiveness of RTS/CTS handshake in \{IEEE 802.11 based ad hoc networks. Ad Hoc Netw. 1(1), 107-123 (2003). doi:10.1016/S1570-8705(03)00015-5

37. J Bicket, D Aguayo, S Biswas, R Morris, in Proceedings of the 11th Annual International Conference on Mobile Computing and Networking, MobiCom. Architecture and evaluation of an unplanned 802.11B mesh network (ACM New York, USA, 2005), pp. 31-42. doi:10.1145/1080829.1080833

38. G Anastasi, M Conti, E Gregori, in Mobile Ad Hoc Networking, ed. by S Basagni, M Conti, S Giordano, and I Stojmenovic. IEEE 802.11 in Ad Hoc networks: protocols, performance and open issues (IEEE Press - John Wiley \& Sons New Jersey, USA, 2004), pp. 69-116

39. R Braden, L Zhang, S Berson, Resource reservation protocol (RSVP) version 1 functional specification. request for comments (RFC) 2205 Internet Engineering Task Force (IETF) (Sept. 1997). Available: http://www. rfc-editor.org/rfc/rfc2205.txt [Accessed 26 May 2014]

40. R Fielding, J Gettys, JC Mogul, H Frystyk, L Masinter, PJ Leach, T BernersLee, Hypertext transfer protocol - HTTP/1.1. Request for Comments (RFC) 2616, Internet Engineering Task Force (IETF) (June 1999). Available: http:// www.rfc-editor.org/rfc/rfc2616.txt [Accessed 28 May 2014]

41. BA Mah, in IEEE Proceedings of 16th Annual Joint Conference of the IEEE Computer and Communications Societies (INFOCOM), vol. 2. An empirical model of HTTP network traffic (Kobe, Japan, 1997), pp. 592-600. doi:10.1109/INFCOM.1997.644510

42. C Park, H Shen, JS Marron, F Hernandez-Campos, D Veitch, in 14th IEEE International Symposium on Modeling, Analysis, and Simulation of Computer and Telecommunication Systems (MASCOTS). Capturing the elusive poissonity in web traffic (Monterey, CA, USA, 2006), pp. 189-196. doi:10.1109/MASCOTS.2006.17

43. I Aktas, T King, C Mengi, in Modeling and Tools for Network Simulation, ed by K Wehrle, M Güneş, and J Gross. Modeling application traffic (Springer Berlin, 2010), pp. 397-426. doi:10.1007/978-3-642-12331-3_18

44. LE Miller, Validation of $802.11 \mathrm{a} /$ UWB coexistence simulation [online] Technical report, National Institute of Standards and Technology, Gaithersburg, MD, USA (Oct. 2003). Available: http://www.antd.nist.gov/ wctg/manet/docs/coexvalid_031017.pdf [Accessed 10 March 2014]

45. G Pei, TR Henderson, Validation of OFDM error rate model in ns-3 [online] (2010). Technical report, Boeing Research \& Technology, Seattle, WA, USA Available: http://www.nsnam.org/ pei/80211 ofdm.pdf [Accessed 10 March 2014]

46. G Lukas, T Lindhorst, E Nett, in 30th IEEE Symposium on Reliable Distributed Systems (SRDS). Modeling medium utilization for admission control in industrial wireless mesh networks (Madrid, Spain, 2011), pp. 65-74. doi:10.1109/SRDS.2011.17

doi:10.1186/1687-1499-2014-219

Cite this article as: Viegas et al:: Real-time communication in IEEE $802.11 \mathrm{~s}$ mesh networks: simulation assessment considering the interference of non-real-time traffic sources. EURASIP Journal on Wireless Communications and Networking 2014 2014:219. 